\title{
HACIA LA RECUPERACIÓN EN LA SALUD MENTAL
}

\section{MIRIAM BRONCANO BOLZONI}

Enfermera asistencial en la Unidad de Hospitalización de Agudos. Institut d'Assistència Sanitària de Girona. Profesora asociada del Departamento de Enfermería. Universitat de Girona. Vicepresidenta de la Associació Catalana d'Infermeria de Salut Mental (ASCISAM).

\section{RESUMEN}

El objetivo de este artículo es abordar los hallazgos fundamentales de la literatura científica referentes a la recuperación en la salud mental, pasando por su definición, algunos de los modelos que la conceptualizan, diferentes instrumentos para medirla y los principios clave para la incorporación de la recuperación en los servicios de salud mental. En la discusión se plantea la recuperación como un cambio de paradigma; se trata de una evolución en el entendimiento mismo de la naturaleza de los trastornos mentales que va más allá de la enfermedad o la superación de los síntomas. La recuperación debe ser un punto de apoyo para el crecimiento y enriquecimiento de la ayuda que se ha de prestar a las personas que padecen un problema de salud mental. El desarrollo de servicios de salud mental basados en la recuperación es una oportunidad, puede marcar el camino hacia dónde nos dirigimos, aunque esto implicaría un profundo cambio en muchos niveles. No obstante, para ello es necesario que los valores clave estén integrados en los usuarios, familias y cuidadores, gestores, promotores, políticos, etc., y poder trabajar de una manera multidisciplinar bajo este paradigma.

Palabras clave: recuperación, salud mental, trastornos mentales, servicios de salud mental.

\section{INTRODUCCIÓN}

La prevalencia y la incidencia de los trastornos mentales es elevada y el impacto que la enfermedad mental tiene a nivel de costes económicos y sociales está documentada en diversos estudios ${ }^{1-4}$. Estos resulta-

Correspondencia: M. Broncano.

Correo electrónico: miriambroncano@gmail.com dos ponen de manifiesto que el modelo de tratamiento centrado en la patología, enfermedad o los síntomas tiene que ir evolucionando, y es necesaria la incorporación de la perspectiva de la recuperación, para acercarnos a la salud, las fortalezas y el bienestar de las personas con trastorno mental.

Para que la recuperación tenga el impacto que merece, los profesionales de la salud necesitamos entender qué significa y, junto con los pacientes y 
otras personas implicadas, respaldar activamente su implementación en los servicios ${ }^{5}$.

Este artículo pretende abordar los hallazgos fundamentales de la literatura científica referentes a la recuperación en la salud mental, pasando por su definición, algunos de los modelos que la conceptualizan, diferentes instrumentos para medirla y los principios clave para la incorporación de la recuperación en los servicios de salud mental.

\section{DEFINICIÓN}

A la idea de «recuperación» le ha llegado su momento ${ }^{6}$. En los últimos años, el término recuperación (recovery en inglés) se ha convertido en una palabra de moda en el ámbito de la salud mental. Prueba de ello son las docenas de veces que se ha definido este concepto 7 . Sin embargo, esto ha ido asociado a una falta de claridad para definir y delimitar este término, ya que no existe una definición universal aceptada. Por otro lado, en la literatura científica, el concepto de recuperación se ha utilizado indistintamente para referirse a modelo, filosofía, enfoque, paradigma, movimiento o visión? ${ }^{8}$.

\section{A la idea de «recuperación» le ha llegado su momento}

Aunque pronósticos médicos predecían un deterioro de la salud mental en personas con esquizofrenia (la teoría de Kraepelin de la esquizofrenia), en la década de 1990, muchos usuarios de los servicios de salud mental desafiaron este camino teórico, describiendo sus experiencias positivas de recuperación. Patricia Deegan fue una de las primeras en escribir acerca de la recuperación como una experiencia transformadora que iba mucho más allá del retorno a la salud' .

William Anthony definió la década de 1990 como la década de la recuperación, y es uno de los funda- dores intelectuales del movimiento de la recuperación. La definió como: «un proceso único, hondamente personal, de cambio de actitudes, valores, sentimientos, metas, habilidades y roles de una persona. Es una manera de vivir una vida satisfactoria, con esperanza y aportaciones, incluso con las limitaciones causadas por la enfermedad. La recuperación implica desarrollar un nuevo sentido y propósito en la vida, a la vez que la persona crece más allá de los efectos catastróficos de la enfermedad mental» ${ }^{10}$.

Otra definición ampliamente utilizada de la recuperación es la de la Substance Abuse and Mental Health Services Administration (SAMHSA) ${ }^{11}$, que se refiere a ella como «un proceso de cambio a través del cual las personas puedan mejorar su salud y bienestar, vivir una vida autónoma, y tratar de alcanzar su pleno potencial». Esta definición describe diez componentes fundamentales: la esperanza, la iniciativa propia, la multiplicidad de senderos, el enfoque integral/holístico, el apoyo de pares, las relaciones humanas, la cultura, la confrontación de trauma, las fortalezas y sentido de responsabilidad y el respeto. La SAMHSA también describe cuatro grandes dimensiones que apoyan una vida en recuperación: la salud, el hogar, el propósito y la comunidad ${ }^{11}$.

Según Shepperd et al., en la base de la recuperación «... reside un conjunto de valores sobre el derecho de una persona a construir por sí misma una vida con sentido, con o sin la continua presencia de los síntomas de una enfermedad mental. La recuperación se basa en los conceptos de autodeterminación y autocontrol. Enfatiza la importancia de la "esperanza" para mantener la motivación y apoyar las expectativas de una vida individual plena» ${ }^{6}$.

Un principio fundamental de la recuperación es que no significa necesariamente una cura (recuperación clínica), sino que enfatiza en el recorrido de una persona que vive con problemas de salud mental, en el proceso de construir su propia vida más allá de la enfermedad mental (recuperación social). Por tanto, una persona puede recuperar su vida sin necesariamente recuperarse de su enfermedad ${ }^{6}$. 
El paradigma de la recuperación representa la convergencia de diferentes ideas (empoderamiento, autogestión, derechos de las personas, inclusión social y rehabilitación), bajo un solo encabezado que señala una nueva dirección para los servicios de salud mental12.

\section{Modelos de recuperación}

A menudo, el modelo de recuperación se ha definido como opuesto al modelo médico. El modelo médico sostiene que la enfermedad mental es crónica, con un pronóstico de disminución del funcionamiento a menos que haya un diagnóstico y tratamiento adecuado. El énfasis está en el poder del experto y el control de la atención de los usuarios. Por el contrario, la recuperación hace hincapié en la creación de una comunidad de empoderamiento donde los usuarios son los expertos de su propio cuidado. El modelo médico se centra en la enfermedad, mientras que la recuperación se centra en el bienestar, las fortalezas y la capacidad de recuperación. Tales visiones del mundo diferentes engendran implícitamente perspectivas culturales dispares.

Ken Wilber intenta crear un marco conceptual que reúna las diferentes posiciones ideológicas para dar forma al paradigma de la recuperación contemporánea. Para ello define el modelo de cuatro cuadrantes, comúnmente denominado $A Q A L$, que significa «todos los cuadrantes, todos los niveles». Este enfoque puede utilizarse como una estrategia para resolver algunas de las dificultades relacionadas con la definición de la recuperación de la salud mental en un contexto contemporáneo. Wilber toma la idea del «yo», «nosotros» y «eso», como dimensiones de la realidad, y las inserta en un modelo de cuatro cuadrantes. Los cuatro cuadrantes presentan un marco para situar varias teorías, junto con sus correspondientes métodos de investigación, en cuatro dominios específicos, con dos ejes (individual/colectiva e interno/externo) de acuerdo con la lente a través de la cual se percibe el mundo. Un paso impor- tante asociado con el enfoque de cuatro cuadrantes de Wilber es reconocer la interrelación de los cuatro dominios de conocimiento ${ }^{13}$.

El modelo de proceso de recuperación propuesto por Green aporta un marco para: a) la comprensión del proceso global de recuperación, b) la identificación de los factores necesarios para la recuperación, y c) el desarrollo de la investigación, las intervenciones y los hábitos de la mente para fomentar la recuperación. Los elementos del escenario para que se desarrolle el proceso de recuperación son: el medio ambiente, los recursos y las tensiones. El aprendizaje, la curación, su manifestación conductual primaria y la adaptación se presentan como su núcleo. Los componentes que facilitan la recuperación incluyen: fuentes de motivación (esperanza, optimismo y sentido), los requisitos previos para la acción (agencia, control y autonomía) y la capacidad (competencia y disfunción) ${ }^{14}$.

Lloyd conceptualiza la recuperación en un marco de seis dimensiones relacionadas entre sí: clínica (la remisión o la reducción de los síntomas), personal/psicológica (la esperanza, el empoderamiento, la actividad significativa, la responsabilidad personal/autodeterminación, la transformación, la espiritualidad, el afrontamiento), cuidado personal (participación significativa en las actividades básicas e instrumentales de cuidado personal), social (la participación significativa en las relaciones y actividades sociales), laboral (la participación significativa en el empleo, la educación, el ocio y otras actividades relacionadas), y del medio ambiente/contexto (alojamiento y apoyos en el entorno físico, político, social y económico del individuo) ${ }^{15}$.

La promoción de la salud y la recuperación de la salud mental parecen haber evolucionado a lo largo de líneas paralelas en una dirección común, sin llegar a converger. Mientras que las cinco estrategias de la Carta de Ottawa son interdependientes, una estrategia no se sostiene sin las otras, y la propia carta está estructurada de tal manera que una estrategia conduce lógicamente a la siguiente. Las super- 
posiciones son inevitables y ciertas estrategias tienen metas y desafíos en común. Del mismo modo, la recuperación es un principio rector y el marco operativo para el sistema de atención, y esta debe colorear todos los servicios existentes, para hacer que las diferentes comunidades, ambientes y entornos sean más propicios para la recuperación, que es implícitamente ecológica. Es decir, la recuperación está relacionada con la promoción de la salud mental a través de la Carta de Ottawa. Se tratan muchos temas y subtemas, y el Modelo Global sugiere hacer el seguimiento de las diversas actividades que se desarrollan en cinco dimensiones: supranacional, estatal, comunitario, organizacional e individual. A cada una de estas dimensiones le corresponde un nivel de análisis y unos medios para promover la recuperación: político, socioeconómico, cultural, interpersonal e intrapersonal. Estos niveles se superponen y entrelazan. En este sentido, el Modelo Global permite la bidireccionalidad y la reciprocidad. Puede ser utilizado para identificar y hacer el seguimiento de las diversas actividades que se desarrollan en las cinco dimensiones de intervención ${ }^{16}$.

El modelo de dos continuos de Keyes incorpora una visión patógena y salutogénica. Desde la perspectiva completa de la salud mental, la recuperación se basa en dos experiencias independientes pero complementarias: la restauración de la enfermedad mental y la optimización de la salud mental positiva, y cada experiencia se define como un proceso y como un resultado, pero complementarios. A través de la recuperación, los individuos tratan de manejar las limitaciones impuestas por la enfermedad mental, mientras se trabaja hacia el alivio de los síntomas y la reducción de la discapacidad, así

\section{El modelo Tidal subraya la importancia de las historias personales del paciente mental y la utilización de su propia voz}

como la curación de las consecuencias sociales negativas de la enfermedad (p. ej., el estigma). Por lo tanto, este modelo no rechaza una concepción patogénica de recuperación, pero lo ve como insuficiente para capturar este fenómeno. Un enfoque salutogénico también es necesario para promover la recuperación, centrándose en el desarrollo óptimo de las fortalezas y otras características de la salud mental positiva ${ }^{17}$.

El modelo Tidal, desarrollado por Phil Barker y Poppy Buchanan-Barker, describe un proceso de cuidados basado en la integración del presente y el futuro a través de tres dimensiones de su personalidad: el dominio del «yo» (necesidad de seguridad emocional y física), el dominio del «mundo» (necesidad de ser comprendido/a y tener validadas sus percepciones), y el dominio de los «otros» (tipo de apoyo que necesita del mundo social para vivir una vida normal). El proceso de cuidados puede empezar en cualquiera de estos tres dominios y el modelo contempla intervenciones individuales y grupales. Este modelo define 10 compromisos basados en valores esenciales, que orientan a la enfermera en el desarrollo de respuestas para ayudar al paciente a cubrir sus necesidades. El modelo es aplicable en entornos de hospitalización breve, donde se inició, y ha sido evaluado en servicios públicos. El modelo Tidal subraya la importancia de las historias personales del paciente mental y la utilización de su propia voz ${ }^{18,19}$

La Recovery Alliance Theory (RAT) o teoría de la alianza y recuperación está basada en la filosofía humanística, que se desarrolló para ser aplicada directamente en la práctica de la enfermería de salud mental. La RAT fue desarrollada en Australia e Irlanda por Eamon Shanley y Maureen Jubb-Shanley, en el año 2000, junto con usuarios, cuidadores familiares y profesionales de la clínica y del entorno académico. Esta teoría está compuesta por seis constructos: la filosofía humanística, la humanidad común, el trabajo conjunto, la recuperación, el enfoque de fuerza y el empoderamiento. De estos constructos 
se derivan tres conceptos: afrontamiento, alianza de trabajo y autorresponsabilidad y control, que forman la base para un sistema de cuidados llamado Partnership in Coping. La construcción de esta teoría se ha inspirado en la preocupación por los derechos humanos, los avances en la autonomía del paciente, la atención comunitaria, el enfoque de la recuperación y la atención centrada en la persona ${ }^{20}$.

\section{Instrumentos}

Uno de los pasos principales para la creación de un sistema sanitario orientado hacia la recuperación es el uso de medidas que permitan evaluar la recuperación de los usuarios, profesionales, programas en particular y servicios, y si el sistema en su conjunto es eficaz en la promoción de la recuperación.

Evaluar un constructo como la recuperación resulta bastante complejo si se tiene en cuenta que es un concepto holístico y personal, para el que existen diversas definiciones y falta de consenso, como se ha comentado anteriormente.
Diversas revisiones han identificado una serie de instrumentos estandarizados que se han desarrollado para tratar de evaluar aspectos de la recuperación en salud mental ${ }^{21-23}$. Por otro lado, a través de nuestra propia estrategia de búsqueda se han identificado otros 5 instrumentos ${ }^{24-28}$, que suman un total de 48 instrumentos. De estos, 27 están diseñados para medir la recuperación de la persona (tabla 1). Estos instrumentos utilizan diversas formas de evaluación: entrevistas (datos cualitativos) o cuestionarios (datos cuantitativos); algunos de ellos evalúan dimensiones de la recuperación, otras actitudes, estilos, procesos o programas específicos. Por otro lado, los 19 instrumentos restantes están diseñados para evaluar la orientación de la recuperación de los servicios (tabla 2); algunos abordan el conocimiento y las actitudes del personal, otros los puntos fuertes y debilidades de los servicios o las prácticas basadas en la recuperación. Hay algunos de los instrumentos que tienen diferentes versiones (usuarios, familiares o cuidadores, proveedores y gestores de los servicios).

Tabla 1. Instrumentos diseñados para evaluar la recuperación de las personas

1. Agreement with Recovery Attitudes Scale 21,22

2. Consumer Recovery Outcomes System ${ }^{21,22}$

3. Crisis Hostel Healing Scale 21,22

4. Illness Management and Recovery Scales ${ }^{21,22}$

5. Maryland Assessment of Recovery Scale 24

6. Mental Health Recovery Measure 21,22

7. Mental Health Recovery Star 21,22

8. Milestones of Recovery Scale 21,22

9. Multi-Phase Recovery Measure 21,22

10. Ohio Mental Health Consumer Outcomes System ${ }^{21,22}$

11. Peer Outcomes Protocol 21,22

12. Personal Vision of Recovery Questionnaire 21,22

13. Questionnaire on the Processes of Recovery ${ }^{21}$

14. Reciprocal Support Scale21,22
15. Recovery Assessment Scale ${ }^{21,22}$

16. Recovery Attitudes Questionnaire 21,22

17. Recovery Elements Assessment Questionnaire-Patient Version 25

18. Recovery Interview ${ }^{21,22}$

19. Recovery Measurement Tool21,22

20. Recovery Orientation ${ }^{21,22}$

21. Recovery Process Inventory21,22

22. Recovery Style Questionnaire ${ }^{26}$

23. Relationships and Activities that Facilitate Recovery Survey ${ }^{21,22}$

24. Rochester Recovery Inquiry 21,22

25. Self-Identified Stage of Recovery ${ }^{21,22}$

26. Stages of Recovery Instrument ${ }^{21,22}$

27. Stages of Recovery Scale ${ }^{27}$ 
Tabla 2. Instrumentos diseñados para evaluar los servicios orientados hacia la recuperación

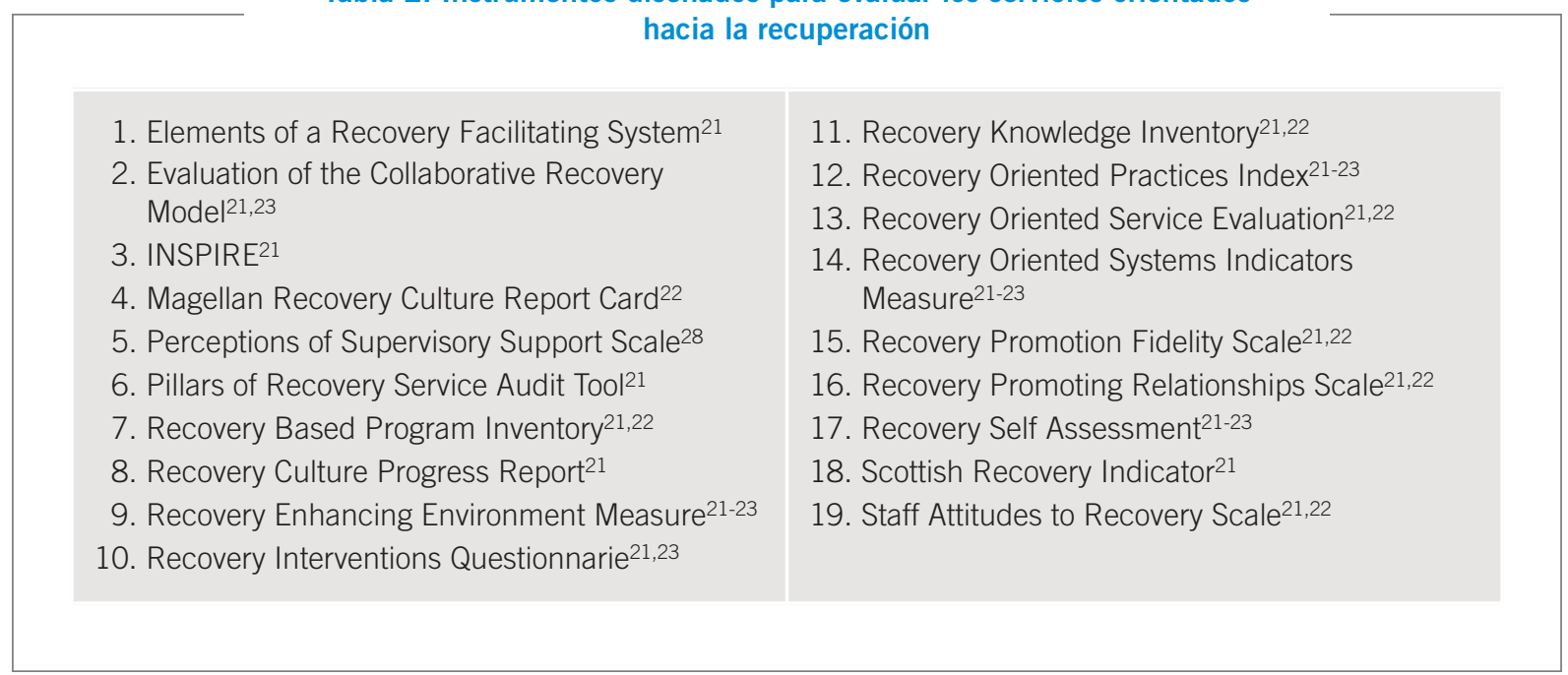

Cabe señalar que únicamente hemos encontrado un instrumento adaptado y validado en población española, el Stage of Recovery Instrument (STORI) ${ }^{29}$. Y la Recovery Self Assessment-Registred Nurse (RSA-RN) que ha sido traducida y adaptada al español ${ }^{5}$.

\section{Servicios orientados a la recuperación}

Tanto los usuarios y sus familias como los profesionales, gestores y políticos están mostrando un interés creciente por la adopción del enfoque de la recuperación como el principio rector de las políticas de salud mental, las prácticas profesionales y la orientación de los servicios prestados. Prueba de ello son las diversas publicaciones e investigaciones que ponen de manifiesto la importancia de incorporar la perspectiva de recuperación en las políticas de salud en lugares como Australia22,30, Nueva York²1, Canadá31, Irlanda ${ }^{32}$, Inglaterra ${ }^{33}$, Escocia ${ }^{34}$ o España ${ }^{35}$.

No obstante, para lograr que los servicios de salud mental se orienten hacia esa concepción de la asistencia, de acompañar a las personas con enfermedad mental en su proceso de recuperación, se requie- re un profundo cambio organizativo en muchos niveles. El Center for Mental Health ha identificado diez retos organizativos clave para la implementación del enfoque de la recuperación en los servicios de salud mental: 1) cambiar la naturaleza de las interacciones diarias y la calidad percibida; 2) impartir programas educativos y formativos integrales liderados por las personas usuarias; 3) constituir un centro de formación sobre recuperación que impulse los programas; 4) garantizar el compromiso organizativo y crear "cultura» de recuperación; 5) aumentar la personalización y la toma de decisiones; 6) cambiar el modo en que enfocamos la evaluación y gestión de riesgos; 7) redefinir la participación de las personas usuarias; 8) transformar al colectivo de profesionales; 9) apoyar a las y los profesionales durante su propia recuperación, y 10) aumentar las posibilidades de construir una vida más allá de la enfermedad ${ }^{12}$.

El enfoque de la recuperación también requiere una relación diferente entre las personas usuarias de los servicios y los profesionales de la salud. La meta del profesional es, por lo tanto, ofrecer a la persona los recursos (información, habilidades, redes y apoyo) que le sirvan para gestionar su propia enfer- 


\section{El enfoque de la recuperación} también requiere una relación diferente entre las personas usuarias de los servicios y los profesionales de la salud

medad en la medida de lo posible y ayudarle a obtener acceso a los recursos que se estimen necesarios para vivir su vida ${ }^{7}$. Los usuarios han de tener un rol activo y voz propia, para poder avanzar en este proceso.

Los objetivos de los servicios de salud mental orientados hacia la recuperación son, por lo tanto, distintos de los objetivos tradicionales de los servicios de salud («tratamiento y cura»). Estos últimos ponen el énfasis en el alivio de síntomas y la prevención de recaídas. En la recuperación, la mejoría de los síntomas sigue siendo importante, y tiene un papel clave en la recuperación de la persona, pero la calidad de vida y el bienestar, según la valore el propio afectado, es fundamental? .

\section{DISCUSIÓN}

La recuperación conlleva un cambio de paradigma. Se trata de una evolución en el entendimiento mismo de la naturaleza de los trastornos mentales que va más allá de la enfermedad o la superación de los síntomas, acercándose a la salud, al bienestar, a las fortalezas y a las relaciones de ayuda. La recuperación debe ser un punto de apoyo para el crecimiento y enriquecimiento de la ayuda que se ha de prestar a las personas que padecen un problema de salud mental. Cabe señalar que actualmente existen diferentes acciones que retoman este enfoque (p. ej., los movimientos de los usuarios en salud mental [el Proyecto Emilia, los Agentes de Ayuda Mutua, etc.] o la existencia de redes [la Red de escuchadores de voces o Red de trabajo de recuperación de Escocia, etc.]), donde se trabaja la ayuda mutua, la lucha contra el estigma o el respeto a los derechos humanos.

El desarrollo de servicios de salud mental basados en la recuperación es una oportunidad que puede marcar el camino hacia donde nos dirigimos, aunque esto implicaría un profundo cambio en muchos niveles, competencias, en nuestra manera de trabajar. Para ello es necesario que los valores clave estén integrados en las personas que padecen un trastorno mental, familias y cuidadores, profesionales, gestores, políticos, etc., y poder trabajar de una manera multidisciplinar bajo este paradigma.

\section{BIBLIOGRAFÍA}

1. Haro JM, Palacín C, Vilagut G, Martínez M, Bernal M, Luque I, et al. Prevalencia de los trastornos mentales y factores asociados: resultados del estudio ESEMeDEspaña. Med Clin (Barc). 2006;126(12):445-51.

2. Rocha KB, Pérez K, Rodríguez-Sanz M, Borrell C, Obiols JE. Prevalencia de problemas de salud mental y su asociación con variables socioeconómicas, de trabajo y salud: resultados de la Encuesta Nacional de Salud de España. Psicothema. 2010;22(3):389-95.

3. Parés-Badell O, Barbaglia G, Jerinic P, Gustavsson A, Salvador-Carulla L, Alonso J. Cost of Disorders of the Brain in Spain. PLoS ONE. 2014;9(8):e105471.

4. Prince M, Patel V, Saxena S, Maj M, Maselko J, Phillips $\mathrm{MR}$, et al. No health without mental health. Lancet. 2007;370(9590):859-77.

5. Shepherd G, Boardman J, Slade M. Making Recovery a Reality. London: Sinsbury Centre for Mental Health; 2008. Traducido por el Servicio Andaluz de Salud. Junta de Andalucía. Disponible en: http://www.1decada4.es/profsalud/documentos/

6. Huizing E, Molino JL, Rigol A, Padilla L, Betolaza E, Serna $L$, et al. Opinión de las enfermeras de salud mental de España sobre la prestación de cuidados a pacientes con trastorno mental basada en el enfoque de recuperación. Biblioteca Lascasas. 2013;9(1). Disponible en: http://www.index-f.com/lascasas/documentos/lc0688.php

7. Onken SJ, Craig CM, Ridgway P, Ralph RO, Cook JA. An analysis of the definitions and elements of recovery: a review of the literature. Psychiatr Rehabil J. 2007; 31(1):9-22.

8. Cleary A, Dowling M. Knowledge and attitudes of mental health professionals in Ireland to the concept of 
recovery in mental health: a questionnaire survey. J Psychiatr Ment Health Nurs. 2009;16(6):539-45.

9. Deegan PE. Recovery: the lived experience of rehabilitation. Psychosoc Rehabil J. 1988;11(4):11-9.

10. Anthony WA. Recovery from mental illness: the guiding vision of the mental health service system in the 1990s. Psychosoc Rehabil J. 1993;16(4):11-23.

11. Substance Abuse and Mental Health Services Administration. SAMHSA's Working Definition of Recovery. Rockville: Department of Health and Human Services; 2012.

12. Shepherd G, Boardman J, Slade M. Implementing recovery. A methodology for organizational change. London: Sinsbury Centre for Mental Health; 2008. Traducido por el Servicio Andaluz de Salud. Junta de Andalucía. Disponible en: http://www.1decada4.es/ profsalud/documentos/

13. Starnino VR. An integral approach to mental health recovery: implications for social work. J Human Behav Soc Environ. 2009;19(7):820-42.

14. Green CA. Fostering recovery from life-transforming mental health disorders: a synthesis and model. Soc Theory Health. 2004;2(4):293-314.

15. Lloyd C, Waghorn G, Williams PL. Conceptualising recovery in mental health rehabilitation. $\mathrm{Br} \mathrm{J}$ Occup Ther. 2008;71(8):321-8.

16. Pelletier JF, Davidson L, Roelandt JL. Citizenship and recovery for everyone: a global model of public mental health. Int J Ment Health Promot. 2009;11(4):45-53.

17. Provencher HL, Keyes CLM. Complete mental health recovery: bridging mental illness with positive mental health. J Public Mental Health. 2011;10(1):57-69.

18. Barker P. The Tidal Model: developing an empowering, person-centred approach to recovery within psychiatric and mental health nursing. J Psychiatr Ment Health Nurs. 2001;8(3):233-40.

19. Barker P, Buchanan-Barker P. The tidal model of mental health recovery and reclamation: application in acute care settings. Issues Ment Health Nurs. 2010; 31(3): 171-80

20. Shanley E, Jubb-Shanley M. The recovery alliance theory of mental health nursing. J Psychiatr Ment Health Nurs. 2007;14(8):734-43.

21. Khanam D, McDonald K, Williams Neils C. Recovery: A Toolkit for Mental Health Providers in New York City. New York: NYC Department of Health and Mental Hygiene; 2013.

22. Burgess P, Pirkis J, Coombs T, Rosen A. Assessing the value of existing recovery measures for routine use in
Australian mental health services. Aust N. Z. J Psychiatry. 2011;45(4):267-80.

23. Williams J, Leamy M, Bird V, Harding C, Larsen J, Le Boutillier $\mathrm{C}$, et al. Measures of the recovery orientation of mental health services: systematic review. Soc Psychiatry Psychiatr Epidemiol. 2012;47(11):1827-35.

24. Drapalski AL, Medoff D, Unick GJ, Velligan DI, Dixon LB, Bellack AS. Assessing recovery of people with serious mental illness: Development of a new scale. Psychiatr Serv. 2012;63(1):48-53.

25. Siu BWM, Ng BFL, Li VCK, Yeung YM, Lee MKL, Leung $\mathrm{AYH}$. Mental health recovery for psychiatric inpatient services: perceived importance of the elements of recovery. East Asian Arch Psychiatry. 2012;22(2):39-48.

26. Drayton M, Birchwood M, Trower P. Early attachment experience and recovery from psychosis. $\mathrm{Br} \mathrm{J}$ Clin Psychol. 1998;37(3):269.84.

27. Song L-Y, Hsu S-T. The development of the stages of recovery scale for persons with persistent mental illness. Res Soc Work Pract. 2011;21(5):572-81.

28. Fukui S, Rapp CA, Goscha R, Marty D, Ezell M. The perceptions of supervisory support scale. Adm Policy Ment Health. 2014;41(3):353-9.

29. Lemos-Giráldez S, García-Álvarez L, Paino M, Fonseca-Pedrero E, Vallina-Fernández O, Vallejo-Seco G, et al. Measuring stages of recovery from psychosis. Compr Psychiatry. 2015;56:51-8.

30. Australian Health Ministers' Advisory Council. A National framework for recovery-oriented mental health services: policy and theory. Australia: Australian Health Ministers' Advisory Council; 2013.

31. Canadian Mental Health Association. Recovery rediscovered: Implications for the Ontario mental health system. Ontario: Canadian Mental Health Association; 2003.

32. Mental Health Commission. A recovery approach within the Irish mental health services. Dublin: Mental Health Commission; 2008.

33. National Institute for Mental Health in England. NIMHE Guiding statement on recovery. England: National Institute for Mental Health in England; 2005.

34. Scottish Executive. Rights, Relationships and Recovery: The Report of the National Review of Mental Health Nursing in Scotland. Edinburg: Scottish Executive; 2006.

35. Ministerio de Sanidad, Política Social e Igualdad. Estrategia en Salud Mental del Sistema Nacional de Salud 2009-2013. Madrid: Ministerio de Sanidad, Política Social e Igualdad Centro de Publicaciones; 2011. 